\title{
Bioinformatics analysis of the molecular mechanism of diffuse intrinsic pontine glioma
}

\author{
LEI DENG, PENGJU XIONG, YUNHUI LUO, XIAO BU, SUOKAI QIAN and WUZHAO ZHONG \\ Department of Neurosurgery, The Great Wall Hospital Affiliated to Nanchang University, \\ 94th Hospital of the People's Liberation Army, Nanchang, Jiangxi 30002, P.R. China
}

Received March 23, 2015; Accepted April 12, 2016

DOI: $10.3892 / \mathrm{ol} .2016 .5024$

\begin{abstract}
The present study aimed to elucidate key molecular mechanisms in the progression of diffuse intrinsic pontine glioma (DIPG). The gene expression profile GSE50021, which consisted of 35 pediatric DIPG samples and 10 normal brain samples, was downloaded from the Gene Expression Omnibus database. The differentially-expressed genes (DEGs) in the pediatric DIPG samples were identified. Gene Ontology (GO), Kyoto Encyclopedia of Genes and Genomes (KEGG) and Reactome pathways of DEGs were enriched and analyzed. The protein-protein interaction (PPI) network of the DEGs was constructed and functional modules of the PPI network were disclosed using ClusterONE. A total of 679 DEGs (454 up- and 225 downregulated) were identified in the pediatric DIPG samples. DEGs were significantly enriched in various GO terms, and KEGG and Reactome pathways. The PPI network of upregulated (153 nodes and 298 connections) and downregulated (71 nodes and 124 connections) DEGs, and two crucial modules, were obtained. Downregulated genes in module 2, such as cholecystokinin (CCK), gastrin (GAST), adenylate cyclase 2 (brain) (ADCY2) and 5-hydroxytryptamine (serotonin) receptor 7 (HTR7), were significantly enriched in the calcium signaling pathway, the neuroactive ligand-receptor interaction pathway and in GO terms, such as the G-protein coupled receptor (GPCR) signaling pathway, while upregulated genes in module 1 were not enriched in any pathways or GO terms. CCK and GAST associated with the GPCR signaling pathway, HTR7 enriched in the neuroactive ligand-receptor interaction, and ADCY2 and HTR7 involved in the calcium signaling pathway may be key mechanisms playing crucial roles in the development and progression of DIPG.
\end{abstract}

Correspondence to: Dr Suokai Qian, Department of Neurosurgery, The Great Wall Hospital Affiliated to Nanchang University, 94th Hospital of the People's Liberation Army, 1028 Jinggangshan Boulevard, Nanchang, Jiangxi 30002, P.R. China

E-mail: suokaiqianskq@163.com

Key words: diffuse intrinsic pontine glioma, differentially-expressed genes, Gene Ontology analysis, pathway analysis, module analysis

\section{Introduction}

Diffuse intrinsic pontine glioma (DIPG) is a fatal malignant pediatric brain tumor and the leading cause of cancer-related mortality in children (1). Although collaborative efforts have been made to improve treatments, survival times have remained static over the past three decades, and the vast majority of children succumb within 2 years of diagnosis (2). Radiotherapy is the standard treatment offering a significant but transient improvement, while chemotherapy has not shown any benefit thus far $(1,3)$. Due to a lack of therapeutic benefits from biopsies and the lack of available tumor material, molecular studies of DIPG have been greatly limited, thus hampering the development of targeted therapies for the disease (4). Therefore, an improved understanding of the molecular mechanism of DIPG is required to develop more specifically therapeutic approaches for this disease.

The biological properties of pediatric DIPG are not identical to those of cerebral high-grade gliomas of adults, which contribute to the failure of the majority of therapeutic trials (3). Analysis of the molecular mechanism of DIPG is useful for developing an effective therapy. Previous studies uncovered the unique genetic composition of this pediatric brain cancer, with nearly $78 \%$ found to possess frequent histone $\mathrm{H} 3$ gene (encoding p.Lys27Met) mutations (5). Additionally, Buczkowicz et al performed genomic analysis of DIPG and revealed a novel recurrent activating type I activin A receptor (ACVR1) mutation (6). Mutations in ACVR1 will increase the expression of activin signaling targets, inhibitor of DNA binding 1 (ID1) and ID2, which are known targets of bone morphogenetic protein (BMP) signaling (7-9). In tumors, BMP signaling drives either a differentiation or proliferation response in glioblastoma stem cells, which is to a certain extent associated with the epigenetic state of the cell $(10,11)$. Contrasting effects are associated with BMP signaling depending on the context. These context-dependent consequences may further precipitate the exclusive association between the ACVR1 mutation and DIPG $(12,13)$. Furthermore, whole-genome profiling of pediatric DIPG performed by Zarghooni et al (14) highlighted the roles of platelet-derived growth factor receptor and poly (ADP-ribose) polymerase in DIPG progression. Despite expanded efforts to investigate the genetic basis of DIPG, to date, the molecular mechanisms of its development and progression remain unclear. 
In a previous study, GSE50021 was used only as expression data for investigating what drives DIPGs via integration of whole-genome sequencing with methylation and copy number profiling (6). There was no systematic and comprehensive analysis for this expression profile. In the current study, microarray analysis was performed for screening the differentially-expressed genes (DEGs) in DIPG samples, then hub genes, as well as the significant modules and pathways underlying the onset and progression of DIPG, were identified using a comprehensive bioinformatics methods. The study aimed to identify candidate genes and associated pathways of DIPG to clarify its molecular mechanisms.

\section{Materials and methods}

Source of data. The GSE50021 microarray expression profile was extracted from the Gene Expression Omnibus (http://www.ncbi.nlm.nih.gov/geo/) database based on the GPL13938 Illumina HumanHT-12 WG-DASL V4.0 expression beadchip platform. The gene expression files were deposited by Buczkowicz et al (6). A total of 35 pediatric DIPG samples and 10 normal brain samples were available for the expression array.

After obtaining the raw data, the robust multiarray average (15) method of the Linear Models for Microarray Data (Limma) package (16) in Bioconductor was used to perform background correction, quartile normalization and probe summarization. Finally, the gene expression matrix of the samples was acquired.

Identification of DEGs. As the DEGs were associated with the development of the disease, a t-test in Limma package (16) was used to identify the DEGs in pediatric DIPG samples compared with the normal controls. All genes with a P-value of $<0.01$ and $\log _{2}$ fold change (FC) $\mid>2.0$ were selected as DEGs for further study.

Functional enrichment analysis. Gene Ontology (GO) (17) analysis is applied for functional studies of large-scale genomic or transcriptomic data. The Kyoto Encyclopedia of Genes and Genomes (KEGG) (18) and Reactome (19) are the major recognized pathway-related databases that record information on how genes are networked in various organisms. A functional enrichment analysis was performed by the Database for Annotation, Visualization and Integrated Discovery (DAVID) (20), which is the online tool for systematically extracting biological meaning and functional annotation behind large gene or protein lists.

$\mathrm{GO}$ categories, including biological process (BP), molecular function (MF), and cellular component (CC), of the identified DEGs were enriched using DAVID. Also, KEGG and Reactome pathway enrichment analyses of the DEGs were applied for the identification of the main functional and metabolic pathways involved in DIPG using DAVID. A P-value of $<0.01$ was chosen as the significant threshold.

Functional annotation of DEGs. TSGene [the tumor suppressor gene (TSG) database] (21) is used for further investigation of the molecular mechanisms of TSGs in cancer, and provides detailed annotations for each TSG, such as transcription factor (TF) regulations. The Tumor-Associated Gene (TAG) (22) database consists of five tables, each of which summarizes attributes for a specific entity associated with the TAG.

Based on the data information of TFs, the functional enrichment of the DEGs for transcriptional regulation was assessed and annotated according to the GO and KEGG pathway annotation terms. In addition, the DEGs were mapped into the TSGene and TAG databases to screen the known oncogene and TSGs.

Protein-protein interaction (PPI) network construction and module selection. The PPI network is increasingly used for identifying the associations between proteins in various organisms. The Search Tool for the Retrieval of Interacting Genes (STRING) (23) is an online database providing experimental and predicted PPI information. Cytoscape (24) is a biological graph visualization tool for integrated models of biomolecular interaction networks. In the present study, the STRING online tool was applied to analyze the PPIs of the DEGs with a combined score of $>0.4$ as the cut-off value, then the PPI networks of the up- and downregulated genes were constructed respectively using Cytoscape software.

In addition, based on connectivity degree, the enriched functional modules of the PPI network were disclosed using the ClusterONE Cytoscape plug-in (25). $\mathrm{P}<0.01$ was chosen as the significance threshold. Furthermore, GO and KEGG pathway enrichment analyses of the DEGs in the significant modules were performed using DAVID to analyze the gene function at the molecular level.

\section{Results}

Identification of DEGs. According to t-test analysis of the DEGs in the pediatric DIPG samples compared with the normal controls, 679 DEGs were recorded, including 454 upregulated and 225 downregulated genes. The results showed that the number of upregulated genes was more than the number of downregulated genes.

Functional enrichment analysis. In the present study, 8 and 6 KEGG pathways corresponding to the up- and downregulated genes, respectively, were enriched using the cutoff criterion. Results of the KEGG pathway analysis showed that the enriched pathways of the upregulated genes included the ribosome, focal adhesion and extracellular matrix-receptor interaction pathways (Table I), while those enriched by the downregulated genes included the calcium signaling, cytokine-cytokine receptor interaction and neuroactive ligand-receptor interaction pathways (Table I).

In addition, based on the analysis of Reactome pathways, it was found that upregulated genes were enriched in 47 pathways, while downregulated genes were enriched in 16 pathways. The enriched Reactome pathways of upregulated genes were mainly involved in translation, metabolism of proteins and folding of actin by CCT/TriC. Downregulated genes were mainly enriched in G-protein coupled receptor (GPCR) ligand binding, signaling by GPCR and class A/1 (rhodopsin-like receptors).

Furthermore, GO terms in different GO categories (BP, MF and CC) were also enriched by the selected DEGs. Results showed that the upregulated genes were significantly associated with cellular membrane organization, membrane organization 
Table I. Enriched KEGG pathways of differentially-expressed genes.

\begin{tabular}{lcc}
\hline KEGG pathways & Gene count & P-value \\
\hline Upregulated genes & & $1.45 \times 10^{-5}$ \\
Ribosome & 12 & $2.21 \times 10^{-5}$ \\
Focal adhesion & 18 & $1.85 \times 10^{-4}$ \\
ECM-receptor interaction & 10 & $3.91 \times 10^{-4}$ \\
Shigellosis & 8 & $1.32 \times 10^{-3}$ \\
Adherens junction & 8 & $4.10 \times 10^{-3}$ \\
Regulation of actin cytoskeleton & 6 & $5.92 \times 10^{-3}$ \\
Pathogenic Escherichia coli infection & 6 & $9.68 \times 10^{-3}$ \\
Colorectal cancer & & $6.10 \times 10^{-4}$ \\
Downregulated genes & 8 & $2.11 \times 10^{-3}$ \\
Calcium signaling pathway & 9 & $2.52 \times 10^{-3}$ \\
Cytokine-cytokine receptor interaction & 9 & $2.84 \times 10^{-3}$ \\
Neuroactive ligand-receptor interaction & 5 & $3.73 \times 10^{-3}$ \\
Dilated cardiomyopathy & 4 & $4.66 \times 10^{-3}$ \\
Arachidonic acid metabolism & 5 & \\
Pancreatic secretion & 5 & \\
\hline
\end{tabular}

Gene count represents the number of genes enriched in the KEGG pathway. KEGG, Kyoto Encylopedia of Genes and Genomes; ECM, extracellular matrix.

and protein targeting to the endoplasmic reticulum (Table II), while the downregulated genes were mainly associated with single-multicellular organismal process, plasma membrane part and neuropeptide hormone activity (Table II).

Functional annotation of DEGs. Based on the TF and TAG data, the results showed that upregulated functional genes contained 20 TFs, including c-abl oncogene 1, non-receptor tyrosine kinase, actin-like $6 \mathrm{~A}$ and aryl hydrocarbon receptor, and 32 TAGs ( 8 oncogenes, 15 TSGs and 9 unknown genes associated with cancer progression). Downregulated functional genes contained 7 TFs, including homeobox A7, MEIS homeobox 2 and nescient helix loop helix 2, and 12 TAGs (3 oncogenes, 5 TSGs and 4 unknown genes associated with cancer progression).

PPI network construction and module selection. According to the STRING data, PPI networks of the upregulated (153 nodes and 298 connections) and downregulated (71 nodes and 124 connections) DEGs were obtained (Fig. 1). Upregulated genes with higher node degree mainly were chaperonin-containing TCP1, subunit 2 ( $\beta$ ) (CCT2), integrin, $\beta 1$ (ITGB1), ITGB4, CD44 molecule (Indian blood group), FYN oncogene-related to SRC, FGR, YES (FYN), and cyclin D1 (CCND1) (Fig. 1A). Downregulated genes with higher node degree were as follows: Adenylate cyclase 2 (brain) (ADCY2), prodynorphin (PDYN), cholecystokinin (CCK) and chemokine (C-C motif) receptor 7 (CCR7) (Fig. 1B). The node degrees were $>10$ for those genes.

Functional module analysis. In the present study, the upregulated genes were enriched in 23 significant modules, while the downregulated genes were enriched in 15 significant modules by ClusterONE. The most significant modules enriched by the up- and downregulated genes, respectively, were module 1 (Fig. 2A) and module 2 (Fig. 2B). Module 1 had 7 nodes and 16 edges, while module 2 had 14 nodes and 48 edges.

Based on the connectivity degree, the hub proteins in module 1 were centrosomal protein $63 \mathrm{kDa}$ (CEP63), CDK5 regulatory subunit associated protein 2 (CDK5RAP2), dynein, cytoplasmic 1, intermediate chain 2 (DYNC1I2), centrin, EF-hand protein, 2 (CETN2) and centrosomal protein $152 \mathrm{kDa}$ (CEP152). The hub proteins in module 2 included ADCY2, CCK, 5-hydroxytryptamine (serotonin) receptor 7 (HTR7), gastrin-releasing peptide, gastrin (GAST) and vasoactive intestinal peptide (VIP).

In addition, the upregulated genes in module 1 were not enriched in KEGG pathways and GO terms, while the downregulated genes in module 2 were significantly enriched in 3 KEGG pathways and 51 GO-BP terms. The pathways enriched by the downregulated genes in module 2 were the calcium signaling, neuroactive ligand-receptor interaction and vascular smooth muscle contraction pathways, and the enriched GO terms mainly were the GPCR signaling pathway, cell surface receptor-linked signal transduction and positive regulation of the homeostatic process.

\section{Discussion}

DIPG represents the largest challenge in the field of pediatric neuro-oncology, with no effective treatment and a near $100 \%$ fatality rate (6). Additionally, a paucity of DIPG tissue is available for molecular studies (26). Thus, the molecular mechanism underlying DIPG progression remains unclear. In the present study, 679 DEGs (454 up- and 225 downregulated) were identified in pediatric DIPG samples compared with 
Table II. Top 15 significantly enriched GO terms of differentially-expressed genes.

\begin{tabular}{|c|c|c|c|}
\hline GO ID & Term & Gene count & P-value \\
\hline \multicolumn{4}{|c|}{ Upregulated genes } \\
\hline GO:0016044 & Cellular membrane organization & 46 & $4.96 \times 10^{-9}$ \\
\hline GO:0061024 & Membrane organization & 46 & $5.42 \times 10^{-9}$ \\
\hline GO:0045047 & Protein targeting to ER & 16 & $1.84 \times 10^{-8}$ \\
\hline GO:0072599 & Establishment of protein localization to ER & 16 & $2.11 \times 10^{-8}$ \\
\hline GO:0016043 & Cellular component organization & 165 & $4.61 \times 10^{-8}$ \\
\hline GO:0005515 & Protein binding & 230 & $1.61 \times 10^{-7}$ \\
\hline GO:0003735 & Structural constituent of ribosome & 15 & $5.03 \times 10^{-6}$ \\
\hline GO:0019900 & Kinase binding & 24 & $7.26 \times 10^{-5}$ \\
\hline GO:0030276 & Clathrin binding & 5 & $9.01 \times 10^{-5}$ \\
\hline GO:0032403 & Protein complex binding & 20 & $1.77 \times 10^{-4}$ \\
\hline GO:0044424 & Intracellular part & 360 & $4.02 \times 10^{-13}$ \\
\hline GO:0005829 & Cytosol & 117 & $1.01 \times 10^{-12}$ \\
\hline GO:0005622 & Intracellular & 361 & $2.00 \times 10^{-12}$ \\
\hline GO:0043226 & Organelle & 325 & $8.83 \times 10^{-12}$ \\
\hline GO:0005737 & Cytoplasm & 291 & $1.22 \times 10^{-11}$ \\
\hline \multicolumn{4}{|c|}{ Downregulated genes } \\
\hline GO:0044707 & Single-multicellular organismal process & 98 & $1.13 \times 10^{-7}$ \\
\hline GO:0032501 & Multicellular organismal process & 99 & $3.81 \times 10^{-7}$ \\
\hline GO:0003008 & System process & 43 & $6.41 \times 10^{-7}$ \\
\hline GO:0050877 & Neurological system process & 35 & $6.61 \times 10^{-7}$ \\
\hline GO:0019226 & Transmission of nerve impulse & 24 & $5.55 \times 10^{-6}$ \\
\hline GO:0044459 & Plasma membrane part & 46 & $1.10 \times 10^{-6}$ \\
\hline GO:0031226 & Intrinsic to plasma membrane & 34 & $3.72 \times 10^{-6}$ \\
\hline GO:0044421 & Extracellular region part & 30 & $1.45 \times 10^{-5}$ \\
\hline GO:0005886 & Plasma membrane & 76 & $2.68 \times 10^{-5}$ \\
\hline GO:0071944 & Cell periphery & 77 & $2.95 \times 10^{-5}$ \\
\hline GO:0005184 & Neuropeptide hormone activity & 5 & $4.34 \times 10^{-6}$ \\
\hline GO:0005179 & Hormone activity & 8 & $4.59 \times 10^{-5}$ \\
\hline GO:0072509 & Divalent inorganic cation transmembrane transporter activity & 9 & $4.67 \times 10^{-5}$ \\
\hline GO:0015085 & Calcium ion transmembrane transporter activity & 8 & $6.66 \times 10^{-5}$ \\
\hline GO:0005262 & Calcium channel activity & 7 & $1.77 \times 10^{-4}$ \\
\hline
\end{tabular}

Gene count represents the number of genes enriched in the GO term. GO ID, Gene Ontology identification; ER, endoplasmic reticulum.

normal controls. Based on the functional modules analysis, the upregulated genes in module 1 were not enriched in any GO terms and pathways, while the downregulated genes in module 2 were significantly enriched in various $\mathrm{GO}$ terms and pathways; for example, CCK and GAST were associated with the GPCR signaling pathway, HTR7 was significantly enriched in neuroactive ligand-receptor interaction, and ADCY2 and HTR7 were enriched in the calcium signaling pathway. Thus, the aforementioned downregulated genes and associated pathways merit further consideration.

GAST and CCK were hub proteins with higher node degrees in module 2. GAST and CCK together constitute a family of homologous peptide hormones, which are potent neurotransmitters in the brain and the periphery (27). GAST has been found to function to endogenously modulate the progression of glioma and the invasion to surrounding structures (28). GAST reduces the invasive abilities of gliomas cells in vitro (28), thus, downregulated GAST may enhance the migratory abilities of glioma cells. In addition, CCK is a gut-brain peptide that has been confirmed to be able to induce mitosis in human gliomas (29). A previous study established that CCK-8s and CCK-33 directly stimulate human glioma cell growth and invasion process (28). Also, CCK is downregulated in glioblastoma compared with normal human brain tissues and may function as a TSG in human glioma (30). By contrast, the present study found that GAST and CCK were significantly associated with GPCR signaling pathway. G protein can couple with and activate adenylate cyclase to increase the concentrations of cyclic adenosine monophosphate (cAMP) through the stimulatory GPCR signaling pathways (31). cAMP/protein kinase A (PKA) pathway activation can induce glioma cell differentiation (32). Previous studies have also confirmed that mature amidated GAST peptides act through the G-protein-coupled CCK-2/GAST receptor, and have been 

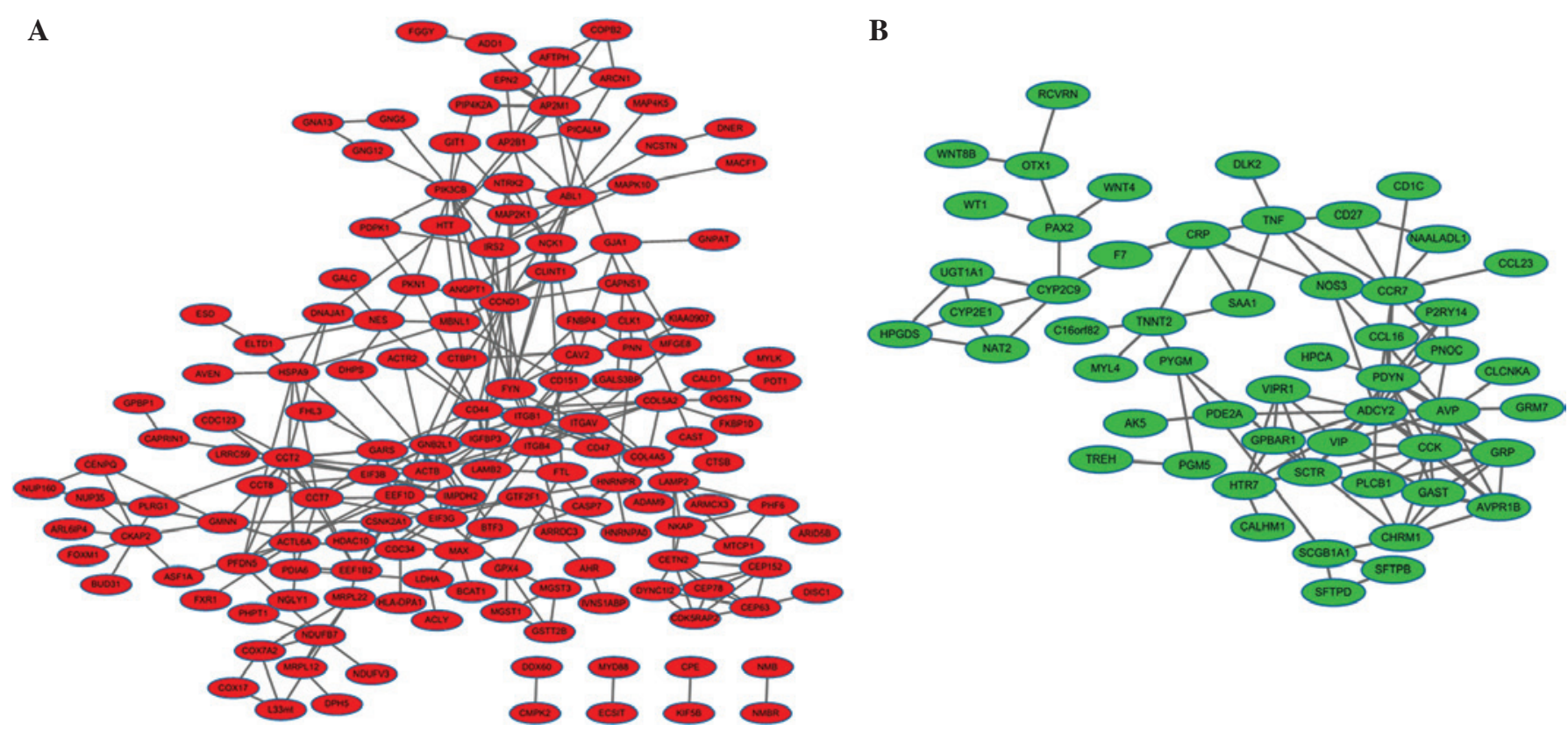

Figure 1. Protein-protein interaction networks of differentially-expressed genes. The red and green nodes indicate (A) upregulated and (B) downregulated genes, respectively. The edges/lines indicate interactions between these genes.

A

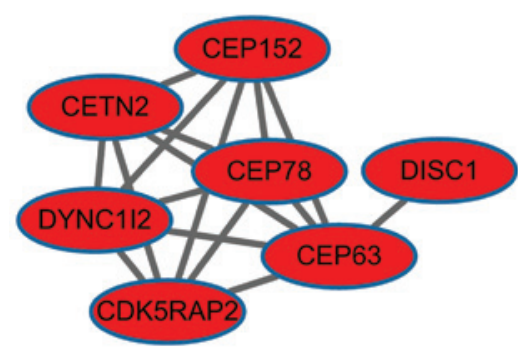

B

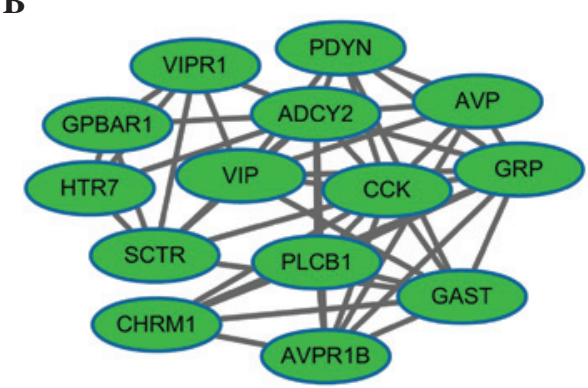

Figure 2. Functional modules of differentially-expressed genes. (A) Module 1 and (B) module 2. The red nodes indicate upregulated genes and green nodes indicate downregulated genes. The edges/lines indicate interactions between these genes.

shown to play a role in malignancy $(33,34)$. Therefore, the present results are in line with previous findings and suggest that GAST and CCK may play roles in DIPG progression via the GPCR signaling pathway.

HTR7 was a hub protein significantly associated with neuroactive ligand-receptor interactions in the present study. The HTR7 gene has been localized to chromosome 10 (10q21-24), and the loss of 10q underpins the progression of DIPG $(35,36)$. Additionally, a study by Chan et al verified that neuroactive ligand-receptor interactions are strongly associated with DIPG tumorigenesis (37). Thus, the present results are consistent with previous findings and suggest that HTR7 may play roles in DIPG tumorigenesis via neuroactive ligand-receptor interactions.

Furthermore, HTR7 was not only enriched in neuroactive ligand-receptor interactions, but also in the calcium signaling pathway. Additionally, ADCY2 was a hub protein significantly associated with the calcium signaling pathway. Calcium is an intracellular signaling molecule participating in tumor cell growth and metastasis (38). Calcium signaling has significant functions in numerous signaling processes in the proliferation and motility of glioblastoma cells (39). Additionally, ADCY2 encodes a member of the family of adenylate cyclases (40) and the activity of HTR7 receptor mediated by G proteins can stimulate adenylate cyclase (41). Adenylate cyclases catalyze the formation of cAMP (42). cAMP can trigger the calcium signaling pathway and be involved in glioma cell differentiation via the cAMP/PKA pathway $(32,43)$. Although the roles of HTR7 and ADCY2 in the progression of DIPG have not been fully discussed, we hypothesize that HTR7 and ADCY2 could play roles in DIPG tumorigenesis via the calcium signaling pathway mediated by cAMP. Further genetic studies are required to confirm this supposition.

Taken together, the results of the present study indicated that CCK and GAST in association with the GPCR signaling pathway, HTR7 enriched in neuroactive ligand-receptor interaction, and ADCY2 and HTR7 involvement in the calcium signaling pathway may be key mechanisms playing crucial roles in the development and progression of DIPG. The present findings provide novel insights into the molecular mechanism of DIPG progression and suggest directions for future research. A better understanding of the molecular mechanism of the pathogenesis of DIPG will provide a broader perspective for further clinical molecular 
target therapy experiments. However, as no experiments were performed in the present study, experimental validation is required to confirm these observations.

\section{References}

1. Hargrave D, Bartels U and Bouffet E: Diffuse brainstem glioma in children: Critical review of clinical trials. Lancet Oncol 7: 241-248, 2006

2. Puget S, Philippe C, Bax DA, Job B, Varlet P, Junier MP, Andreiuolo F, Carvalho D, Reis R, Guerrini-Rousseau L, et al: Mesenchymal transition and PDGFRA amplification/mutation are key distinct oncogenic events in pediatric diffuse intrinsic pontine gliomas. PLoS One 7: e30313, 2012.

3. Donaldson SS, Laningham F and Fisher PG: Advances toward an understanding of brainstem gliomas. J Clin Oncol 24: 1266-1272, 2006.

4. Paugh BS, Broniscer A, Qu C, Miller CP, Zhang J, Tatevossian RG, Olson JM, Geyer JR, Chi SN, da Silva NS, et al: Genome-wide analyses identify recurrent amplifications of receptor tyrosine kinases and cell-cycle regulatory genes in diffuse intrinsic pontine glioma. J Clin Oncol 29: 3999-4006, 2011

5. Wu G, Broniscer A, McEachron TA, Lu C, Paugh BS, Becksfort J, Qu C, Ding L, Huether R, Parker M, et al; Project SJCsRHWUPCG: Somatic histone H3 alterations in pediatric diffuse intrinsic pontine gliomas and non-brainstem glioblastomas. Nat Genet 44: 251-253, 2012.

6. Buczkowicz P, Hoeman C, Rakopoulos P, Pajovic S, Letourneau L, Dzamba M, Morrison A, Lewis P, Bouffet E, Bartels U, et al: Genomic analysis of diffuse intrinsic pontine gliomas identifies three molecular subgroups and recurrent activating ACVR1 mutations. Nat Genet 46: 451-456, 2014.

7. Kurooka H, Nakahiro T, Mori K, Sano K and Yokota Y: BMP signaling is responsible for serum-induced Id2 expression. Biochem Biophys Res Commun 420: 281-287, 2012.

8. Kowanetz M, Valcourt U, Bergström R, Heldin $\mathrm{CH}$ and Moustakas A: Id 2 and Id3 define the potency of cell proliferation and differentiation responses to transforming growth factor beta and bone morphogenetic protein. Mol Cell Biol 24: 4241-4254, 2004.

9. Shin M, Ohte S, Fukuda T, Sasanuma H, Yoneyama K Kokabu S, Miyamoto A, Tsukamoto S, Hohjoh H, Jimi E and Katagiri T: Identification of a novel bone morphogenetic protein (BMP)-inducible transcript, BMP-inducible transcript-1, by utilizing the conserved BMP-responsive elements in the Id genes. J Bone Miner Metab 31: 34-43, 2013.

10. Piccirillo S, Reynolds B, Zanetti N, Lamorte G, Binda E, Broggi G, Brem H, Olivi A, Dimeco F and Vescovi AL: Bone morphogenetic proteins inhibit the tumorigenic potential of human brain tumour-initiating cells. Nature 444: 761-765, 2006.

11. Lee J, Son MJ, Woolard K, Donin NM, Li A, Cheng CH, Kotliarova S, Kotliarov Y, Walling J, Ahn S, et al: Epigenetic-mediated dysfunction of the bone morphogenetic protein pathway inhibits differentiation of glioblastoma-initiating cells. Cancer Cell 13: 69-80, 2008.

12. Wu G, Diaz AK, Paugh BS, Rankin SL, Ju B, Li Y, Zhu X, Qu C, Chen X, Zhang J, et al; St. Jude Children's Research Hospital - Washington University Pediatric Cancer Genome Project: The genomic landscape of diffuse intrinsic pontine glioma and pediatric non-brainstem high-grade glioma. Nat Genet 46: 444-450, 2014.

13. Taylor KR, Mackay A, Truffaux N, Butterfield YS, Morozova O, Philippe C, Castel D, Grasso CS, Vinci M, Carvalho D, et al: Recurrent activating ACVR1 mutations in diffuse intrinsic pontine glioma. Nat Genet 46: 457-461, 2014.

14. Zarghooni M, Bartels U, Lee E, Buczkowicz P, Morrison A, Huang A, Bouffet E and Hawkins C: Whole-genome profiling of pediatric diffuse intrinsic pontine gliomas highlights platelet-derived growth factor receptor alpha and poly (ADP-ribose) polymerase as potential therapeutic targets. J Clin Oncol 28: 1337-1344, 2010.

15. Irizarry RA, Hobbs B, Collin F, Beazer-Barclay YD, Antonellis KJ, Scherf U and Speed TP: Exploration, normalization, and summaries of high density oligonucleotide array probe level data. Biostatistics 4: 249-264, 2003.

16. Smyth GK: Limma: Linear models for microarray data. In: Bioinformatics and Computational Biology Solutions using R and Bioconductor. Gentleman R, Carey VJ, Huber W, Irizarry RA and Dudoit S (eds). Springer-Verlag, New York, pp397-420, 2005
17. Ashburner M, Ball CA, Blake JA, Botstein D, Butler H, Cherry JM, Davis AP, Dolinski K, Dwight SS, Eppig JT, et al: Gene ontology: Tool for the unification of biology. The gene ontology constortium. Nat Genet 25: 25-29, 2000.

18. Kanehisa M and Goto S: KEGG: Kyoto encyclopedia of genes and genomes. Nucleic Acids Res 28: 27-30, 2000.

19. Croft D, O'Kelly G, Wu G, Haw R, Gillespie M, Matthews L, Caudy M, Garapati P, Gopinath G, Jassal B, et al: Reactome: A database of reactions, pathways and biological processes. Nucleic Acids Res 39 (Database Issue): D691-D697, 2011.

20. Huang da W, Sherman BT and Lempicki RA: Systematic and integrative analysis of large gene lists using DAVID bioinformatics resources. Nat Protoc 4: 44-57, 2009.

21. Zhao M, Sun J and Zhao Z: TSGene: A web resource for tumor suppressor genes. Nucleic Acids Res 41 (Database Issue): D970-D976, 2013.

22. Chen JS, Hung WS, Chan HH, Tsai SJ and Sun HS: In silico identification of oncogenic potential of fyn-related kinase in hepatocellular carcinoma. Bioinformatics 29: 420-427, 2013.

23. Franceschini A, Szklarczyk D, Frankild S, Kuhn M, Simonovic M, Roth A, Lin J, Minguez P, Bork P, von Mering C and Jensen LJ: STRING v9. 1: Protein-protein interaction networks, with increased coverage and integration. Nucleic Acids Res 41 (Database Issue): D808-D815, 2013

24. Smoot ME, Ono K, Ruscheinski J, Wang PL and Ideker T: Cytoscape 2.8: New features for data integration and network visualization. Bioinformatics 27: 431-432, 2011.

25. Maraziotis IA, Dimitrakopoulou K and Bezerianos A: An in silico method for detecting overlapping functional modules from composite biological networks. BMC Syst Biol 2: 93, 2008.

26. Saratsis AM, Yadavilli S, Magge S, Rood BR, Perez J, Hill DA, Hwang E, Kilburn L, Packer RJ and Nazarian J: Insights into pediatric diffuse intrinsic pontine glioma through proteomic analysis of cerebrospinal fluid. Neuro Oncol 14: 547-560, 2012.

27. Rehfeld JF, Friis-Hansen L, Goetze JP and Hansen TV: The biology of cholecystokinin and gastrin peptides. Curr Top Med Chem 7: 1154-1165, 2007.

28. Lefranc F, Camby I, Belot N, Bruyneel E, Chaboteaux C, Brotchi J, Mareel M, Salmon I and Kiss R: Gastrin significantly modifies the migratory abilities of experimental glioma cells. Lab Invest 82: 1241-1252, 2002.

29. Oikonomou E, Buchfelder M and Adams EF: Cholecystokinin (CCK) and CCK receptor expression by human gliomas: Evidence for an autocrine/paracrine stimulatory loop. Neuropeptides 42: 255-265, 2008 .

30. Dmitrenko V, Bojko O, Shostak K, Vitak NY, Bukreeva T, Rozumenko V, Malysheva T, Shamayev M, Zozulya YP and Kavsan VM: Characterization of genes, down-regulated in human glioma, potential tumor suppressor genes. Biopolym Cell 23: 347-362, 2007.

31. Harmar AJ: Family-B G-protein-coupled receptors. Genome Biol 2: REVIEWS3013, 2001

32. Shu M, Zhou Y, Zhu W, Zhang H, Wu S, Chen J and Yan G: MicroRNA 335 is required for differentiation of malignant glioma cells induced by activation of cAMP/protein kinase A pathway. Mol Pharmacol 81: 292-298, 2012.

33. Smith AM and Watson SA: Gastrin and gastrin receptor activation: An early event in the adenoma-carcinoma sequence. Gut 47: 820-824, 2000.

34. Paillasse MR, de Medina P, Amouroux G, Mhamdi L, Poirot M and Silvente-Poirot S: Signaling through cholesterol esterification: A new pathway for the cholecystokinin 2 receptor involved in cell growth and invasion. J Lipid Res 50: 2203-2211, 2009.

35. Oswald P, Souery D and Mendlewicz J: Molecular genetics of affective disorders. Int J Neuropsychopharmacol 6: 155-169, 2003

36. Jones C, Perryman L and Hargrave D: Paediatric and adult malignant glioma: Close relatives or distant cousins? Nat Rev Clin Oncol 9: 400-413, 2012.

37. Chan KM, Fang D, Gan H, Hashizume R, Yu C, Schroeder M, Gupta N, Mueller S, James CD, Jenkins R, et al: The histone H3.3K27M mutation in pediatric glioma reprograms H3K27 methylation and gene expression. Genes Dev 27: 985-990, 2013.

38. Monteith GR, McAndrew D, Faddy HM and Roberts-Thomson SJ: Calcium and cancer: Targeting $\mathrm{Ca} 2+$ transport. Nat Rev Cancer 7: 519-530, 2007

39. Kang SS,Han KS, Ku BM,Lee YK, Hong J, Shin HY,Almonte AG, Woo DH, Brat DJ, Hwang EM, et al: Caffeine-mediated inhibition of calcium release channel inositol 1,4,5-trisphosphate receptor subtype 3 blocks glioblastoma invasion and extends survival. Cancer Res 70: 1173-1183, 2010. 
40. Ludwig MG and Seuwen K: Characterization of the human adenylyl cyclase gene family: cDNA, gene structure, and tissue distribution of the nine isoforms. J Recept Signal Transduct Res 22: 79-110, 2002.

41. Hagan JJ, Price GW, Jeffrey P, Deeks NJ, Stean T, Piper D, Smith MI, Upton N, Medhurst AD, Middlemiss DN, et al: Characterization of SB-269970-A, a selective 5-HT(7) receptor antagonist. Br J Pharmacol 130: 539-548, 2000.
42. Zhang HT, Huang Y, Jin SL, Frith SA, Suvarna N, Conti M and O'Donnell JM: Antidepressant-like profile and reduced sensitivity to rolipram in mice deficient in the PDE4D phosphodiesterase enzyme. Neuropsychopharmacology 27: 587-595, 2002.

43. Schmidt M, Evellin S, Weernink PA, von Dorp F, Rehmann H, Lomasney JW and Jakobs KH: A new phospholipase-C-calcium signalling pathway mediated by cyclic AMP and a Rap GTPase. Nat Cell Biol 3: 1020-1024, 2001. 